\title{
Noninfectious Ascending Aortitis: Staying Ahead of the Curve
}

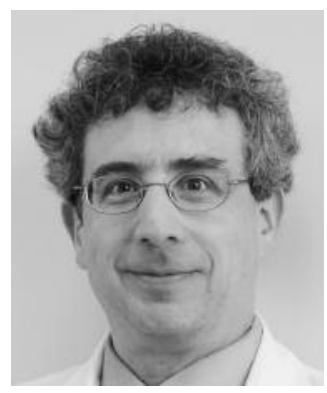

Aortitis is the general name for a spectrum of disorders involving inflammation of the aorta ${ }^{1}$. The causes of aortitis are most easily sorted into either infectious or noninfectious disease. Infections associated with aortitis include syphilis, Salmonella, Staphylococcus, other bacteria, and mycobacteria, especially $M$. tuberculosis. Noninfectious causes of aortitis include several forms of primary and secondary large-vessel vasculitis. Giant cell arteritis (GCA) and Takayasu's arteritis are the most common causes of noninfectious aortitis. At least $20 \%$ of patients with GCA and $50 \%-70 \%$ of patients with Takayasu's arteritis will develop changes consistent with aortitis, many of whom develop aortic aneurysms ${ }^{2-7}$. Aortic involvement is also seen in relapsing polychondritis, Cogan's syndrome, and Behçet's disease, and has been reported as a rare association with other vasculitides and systemic rheumatic diseases including rheumatoid arthritis and spondyloarthropathies. There is growing recognition that "isolated" aortitis may occur without evidence of an infection or systemic disease.

Although patients with Takayasu's arteritis, and increasingly those with GCA, are now routinely screened for aortic involvement, aortitis is still often diagnosed only after surgical specimens taken during repairs of aortic aneurysms or dissections are examined histologically. It is likely that there are many people with aortitis whose aortic lesions never come to medical attention, although the routine use of computed tomography (CT) and magnetic resonance (MR) imaging of the thorax and abdomen for evaluation of many medical conditions will likely lead to a steady increase in the number of cases of "incidental" aortic disease discovered. Furthermore, because many signs and symptoms of large-vessel vasculitis may be vague or simply unrecognized as due to vasculitis, retrospective studies almost certainly underestimate the prevalence of preexisting vasculitis among patients with "incidentally" discovered aortitis.

The clinical effects of aortitis range from asymptomatic mild aortic thickening, stenosis, or aneurysmal dilatation to life-threatening aortic dissection, stenosis, aneurysm, or branch arterial stenosis or occlusion of coronary, renal, mesenteric, or other arteries. Although the development of aortic aneurysms is common with aortitis, the vast majority of aortic aneurysms, both thoracic and abdominal, are due to noninflammatory disease including cystic medial degeneration, atherosclerosis, inherited connective tissue diseases, or a combination of these processes ${ }^{8}$.

The most common serious clinical sequelae of aortitis are thoracic aortic aneurysms, especially of the ascending aorta. The rate of aortic aneurysms among patients with aortitis is markedly elevated compared to the general population, especially for ascending thoracic lesions. The reason for this differential tropism for the proximal versus distal aorta in aortitis, which is the opposite of what is seen in noninflammatory aortic aneurysms, is not clear but may be related to differences in the tissue structure, presence of vasa vasorum, blood flow dynamics, and other factors 9 . Furthermore, why some instances of aortitis lead to aneurysm formation and others to stenosis is also not clear, but these 2 outcomes can occur in the same patient in different areas.

There are 3 basic clinical scenarios in which noninfectious aortitis comes to clinical attention: (1) in patients with an established diagnosis of a systemic condition associated with aortitis; (2) incidentally discovered aortitis with a subsequent diagnosis of an associated systemic disease; and (3) isolated aortitis. The natural histories, evaluations, and management strategies for these 3 scenarios can differ substantially. When reviewing the literature on aortitis it is important to recognize a bias towards descriptions of cases of aortic aneurysms requiring surgical repair, rather than aortitis that results in stenosis of the aorta, asymptomatic aortic thickening, or smaller, non-critical aneurysms.

Most of the current knowledge about noninfectious aortitis stems from published single-institution, retrospective case series of aortitis incidentally discovered on pathologic examination of surgical specimens ${ }^{10-14}$, from cohorts of patients with established large-vessel vasculitis, i.e., Takayasu's arteritis or GCA ${ }^{6,15}$, or from autopsy studies ${ }^{2,14}$. Among the studies focusing on inflammatory disease of the aorta, the great majority of cases involved the thoracic aorta, mostly the ascending portion. The reason for surgery

See Noninfectious ascending aortitis, page 2290

Personal non-commercial use only. The Journal of Rheumatology Copyright $@$ $~ 2009$. All rights reserved. 
in most patients in these series was for repair of ascending aortic aneurysms; aortic valve insufficiency was present in a minority of cases.

The extent of clinical, histological, and imaging data varies widely among studies of aortitis. Further complicating direct comparisons of the series is the use of differing definitions of Takayasu's or GCA, ranging from combinations of age and pathologic findings to fulfillment of published classification criteria. Despite the variability of the data, some fairly consistent findings about noninfectious aortitis have emerged from these studies.

The frequency of noninfectious aortitis among recent series of patients undergoing repair of aortic aneurysms ranged from $1.7 \%$ to $8.8 \%$, with higher frequencies seen when the cases were restricted to disease of the thoracic aorta $^{10-14}$. The age range for noninfectious aortitis is wide (16-85 yrs) with a mean age of roughly 65 years, indicating a skew towards older patients. Most series report a female predominance among patients with aortitis, and this finding is in contrast to the male predominance among patients with noninflammatory aortic disease. The frequency of finding associated systemic inflammatory/autoimmune conditions among patients with aortitis ranges widely, from 5\% to $49 \%$, with such associations likely dependent on the extent of surveillance conducted at the time aortitis was diagnosed, the disease definitions, and institutional referral patterns ${ }^{10,12-14}$. Although routine use of CT or MR angiography will likely increase the discovery rate for associated disease in aortitis, the majority of patients with noninfectious aortitis appear to have "isolated aortitis." Giant cells are commonly seen in aortitis, but their presence does not help to differentiate among cases of isolated aortitis, GCA, or Takayasu's arteritis. Similarly, testing for elevated levels of circulating acute-phase reactants, such as erythrocyte sedimentation rate or C-reactive protein, is not particularly helpful in discerning the etiology or extent of aortitis.

Information on the clinical outcomes of patients with noninfectious aortitis undergoing surgical repair of aneurysms is complicated by incomplete followup and lack of systematic surveillance for new complications of vasculitis. New thoracic or abdominal aneurysms do occur in patients with both isolated aortitis and aortitis associated with systemic vasculitis, although such progression occurs in the minority of patients. The data to date indicate that the longterm outcomes of patients shown to have "isolated" aortitis is generally good, regardless of treatment with glucocorticoids. Furthermore, the efficacy of treating patients with known vasculitis with glucocorticoids to prevent new aortic complications has also not been demonstrated.

The report on noninfectious aortitis by Liang and colleagues in this issue of The Journal both confirms conclusions from prior reports and provides new insights into this disease spectrum ${ }^{16}$. This group reports on a series of patients with noninfectious aortitis of the ascending aorta seen at the
Mayo Clinic between 2000 and 2006. Clinical data were collected retrospectively, but new pathologic review of all aortic specimens was undertaken by a single cardiovascular pathologist. Sixty-four cases of noninfectious aortitis were identified from a total of 766 patients undergoing surgical repair of ascending thoracic aneurysms (frequency of aortitis $=8.4 \%$ ). Half the patients were women and the mean age was 69.1 years. Only $19 \%$ of patients had a history of a systemic rheumatic disease associated with development of aortitis.

A major strength of this new report is that vascular imaging data were available on $89 \%$ of patients; this imaging demonstrated that a remarkable $72 \%$ had vascular abnormalities beyond the ascending aorta, especially additional aortic aneurysms. While some of these abnormalities were certainly due to hypertensive and atherosclerotic disease, a subset was likely due to additional areas of large-vessel vasculitis, which implies that the true incidence of "isolated" aortitis in this cohort is lower than $81 \%$.

The mean time of followup on the new Mayo cohort was only 15.4 months and thus this study provides limited new data on clinical outcomes. However, longer-term followup of such cohorts, cared for at major medical centers under current medical practice, will provide important data to guide clinical management.

The study by Liang and colleagues, combined with the prior cohort reports, provides enough information to develop some general guidelines for clinical evaluation and management of patients discovered to have noninfectious aortitis. Of course, the proper diagnosis of noninfectious aortitis assumes that syphilis and other infections, while now rare causes of aneurysms, are ruled out in all cases. Additionally, it should now be standard practice that surgical specimens from all cases of aortic aneurysm repair be reviewed by a pathologist and the possibility of aortitis be considered.

Patients with newly discovered noninfectious aortitis should be evaluated for both additional areas of arterial disease and signs and symptoms of systemic inflammatory diseases associated with large-vessel vasculitis. Rheumatologists are the appropriate specialists to lead such evaluations, which must include a comprehensive review of medical records, a full patient interview, a complete physical examination with particular focus on the vascular system, and appropriate laboratory testing. If not already done preoperatively, patients should have angiographic studies (MR or CT) of the entire aorta and its main branches to determine if additional arterial lesions consistent with vasculitis are present. Differentiating vasculitic from atherosclerotic lesions can be difficult, especially in older patients or others at increased risk for atherosclerosis. It is key to understand which lesions represent "old" disease and which are new lesions in new arterial territories.

The choice of various imaging modalities for large-vessel vasculitis and the utility of the imaging data both diag-

Personal non-commercial use only. The Journal of Rheumatology Copyright @ $\odot 2009$. All rights reserved. 
nostically and as markers of disease activity remain another area of uncertainty ${ }^{17,18}$. Nonetheless, since one cannot rely on symptoms, physical examination findings, or inflammatory markers to fully assess disease activity in large-vessel vasculitis, some form of serial imaging is a critical component in management and should be conducted in all patients with aortitis.

The main clinical question regarding the assessment and management of "isolated" aortitis still remains: which patients with aortitis require treatment? Given the generally good outcome for patients with aortitis who survive the immediate postoperative period, it is important to avoid overtreatment. Current evidence suggests that it is reasonable to not initiate treatment for patients with isolated aortitis for whom comprehensive evaluation has not revealed evidence of other aortic/branch disease or an associated systemic disease. However, if patients with "isolated" aortitis later develop new lesions felt to be due to inflammatory disease, then such patients should be reclassified as having systemic vasculitis and initiation of medical treatment considered.

If a patient with noninfectious aortitis is found to have a systemic vasculitis or another associated condition, then consideration should be given for some form of antiinflammatory and/or chronic immunosuppressive therapy, depending on the estimated risk of developing new lesions. The decision to go forward with treatment is even more compelling for patients with GCA, Takayasu's arteritis, or other systemic diseases in whom it is clear that there has been recent worsening of aortitis, especially new arterial lesions, including either aneurysms or stenoses. However, it must be made clear to patients that the efficacy of current treatments in preventing new lesions even in established large-vessel vasculitis is not certain. Furthermore, it is important to realize that not all new arterial lesions, even aortic aneurysms, will necessarily become clinically significant for an individual patient. Our current ability to determine which lesions and which patients require chronic therapy for large-vessel vasculitis is quite limited.

All patients with aortitis should be followed for the development of new arterial disease. Regular review of systems and physical examinations should be complemented by screening imaging studies, the type and interval of which is not certain, but annual evaluation of some sort seems a reasonable minimal standard. Regardless of whether or not treatment is directed at aortitis per se, all patients should be routinely assessed for common cardiovascular risks and such risks addressed, especially hypertension.

The data on noninfectious aortitis also provide a rationale to reconsider the currently employed classification and diagnostic criteria used for large-vessel vasculitis. The emerging concept of "isolated" aortitis as an entity distinct from other forms of large-vessel vasculitis with different clinical outcomes means that differentiation of this syn- drome from GCA and Takayasu's arteritis is important. Furthermore, the recognition that aortic involvement in GCA is more common than previously appreciated, combined with increased radiographic surveillance of patients with GCA, makes differentiation of aortitis from other causes of aneurysm increasingly relevant. Some authors have advocated for greater incorporation of histological findings in classifying and diagnosing aortitis, including inflammatory cell type, degree of necrosis, and location of infiltrate ${ }^{14}$. An international group of investigators has begun the process of reconsidering current classification criteria in vasculitis and developing diagnostic criteria ${ }^{19}$. These efforts should lead to new data-driven approaches to include the combination of demographic, clinical, radiographic, laboratory, and pathologic information into a new, clinically meaningful system of differentiating among subsets of patients with noninfectious aortitis.

Rheumatologists need to partner with cardiologists, vascular surgeons, and radiologists to provide coordinated care for all patients with aortitis. The continued study of noninfectious aortitis should lead to improved guidelines for treatment and management as well as a deeper understanding of the pathophysiology of this fascinating disease spectrum.

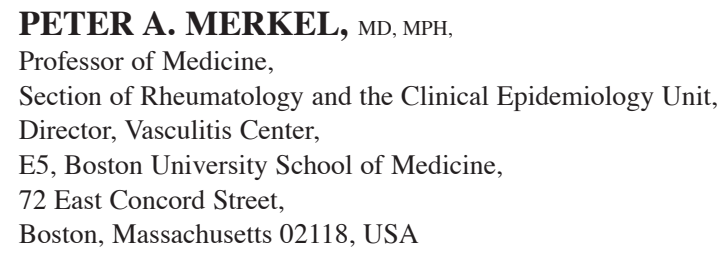

Address correspondence to Dr.Merkel.E-mail:pmerkel@bu.edu

\section{REFERENCES}

1. Gornik HL, Creager MA. Aortitis. Circulation 2008;117:3039-51.

2. Ostberg G. Morphological changes in the large arteries in polymyalgia arteritica. Acta Med Scand Suppl 1972;533:135-59.

3. Klein RG, Hunder GG, Stanson AW, Sheps SG. Large artery involvement in giant cell (temporal) arteritis. Ann Intern Med 1975;83:806-12.

4. Evans JM, O'Fallon WM, Hunder GG. Increased incidence of aortic aneurysm and dissection in giant cell (temporal) arteritis. A population-based study. Ann Intern Med 1995;122:502-7.

5. Nuenninghoff DM, Hunder GG, Christianson TJ, McClelland RL, Matteson EL. Incidence and predictors of large-artery complication (aortic aneurysm, aortic dissection, and/or large-artery stenosis) in patients with giant cell arteritis: a population-based study over 50 years. Arthritis Rheum 2003;48:3522-31.

6. Maksimowicz-McKinnon K, Clark TM, Hoffman GS. Limitations of therapy and a guarded prognosis in an American cohort of Takayasu arteritis patients. Arthritis Rheum 2007;56:1000-9.

7. Garcia-Martinez A, Hernandez-Rodriguez J, Arguis P, Paredes P, Segarra M, Lozano E, et al. Development of aortic aneurysm/ dilatation during the followup of patients with giant cell arteritis: a cross-sectional screening of fifty-four prospectively followed patients. Arthritis Rheum 2008;59:422-30.

8. Homme JL, Aubry MC, Edwards WD, Bagniewski SM, Shane Pankratz V, Kral CA, et al. Surgical pathology of the ascending aorta: a clinicopathologic study of 513 cases. Am J Surg Pathol Personal non-commercial use only. The Journal of Rheumatology Copyright $\subset$ $~ 2009$. All rights reserved. 
2006;30:1159-68

9. Hoffman GS. Large-vessel vasculitis: unresolved issues. Arthritis Rheum 2003;48:2406-14.

10. Rojo-Leyva F, Ratliff NB, Cosgrove DM 3rd, Hoffman GS. Study of 52 patients with idiopathic aortitis from a cohort of 1,204 surgical cases. Arthritis Rheum 2000;43:901-7.

11. Kerr LD, Chang YJ, Spiera H, Fallon JT. Occult active giant cell aortitis necessitating surgical repair. J Thorac Cardiovasc Surg 2000;120:813-5.

12. Miller DV, Isotalo PA, Weyand CM, Edwards WD, Aubry MC, Tazelaar HD. Surgical pathology of noninfectious ascending aortitis: a study of 45 cases with emphasis on an isolated variant. Am J Surg Pathol 2006;30:1150-8

13. Pacini D, Leone O, Turci S, Camurri N, Giunchi F, Martinelli GN, et al. Incidence, etiology, histologic findings, and course of thoracic inflammatory aortopathies. Ann Thorac Surg 2008;86:1518-23.

14. Burke AP, Tavora F, Narula N, Tomaszewski JE, Virmani R. Aortitis and ascending aortic aneurysm: description of 52 cases and proposal of a histologic classification. Hum Pathol 2008;39:514-26.
15. Lie JT. Aortic and extracranial large vessel giant cell arteritis: a review of 72 cases with histopathologic documentation. Semin Arthritis Rheum 1995;24:422-31.

16. Liang K, Chowdhary V, Michet C Jr, Miller D, Sundt T, Connolly H, et al. Noninfectious ascending aortitis: A case series of 64 patients. J Rheumatol 2009;36:2290-7.

17. Kissin EY, Merkel PA. Diagnostic imaging in Takayasu arteritis. Curr Opin Rheumatol 2004;16:31-7.

18. Pipitone N, Versari A, Salvarani C. Role of imaging studies in the diagnosis and follow-up of large-vessel vasculitis: an update. Rheumatology 2008;47:403-8.

19. Basu N, Watts R, Bajema I, et al. EULAR/ACR endorsed points to consider in the development of definitions and classification criteria in the systemic vasculitides. Submitted for publication.

J Rheumatol 2009;36:2137-40; doi:10.3899/jrheum.090920 